\title{
Thin films of silica nanoparticle doped nematic liquid crystal 7CB for electro-optic modulation
}

\author{
Y.G. Marinov, ${ }^{1}$ G.B. Hadjichristov, ${ }^{* 1}$ A.G. Petrov, ${ }^{1}$ and S. Krishna Prasad ${ }^{2}$ \\ ${ }^{1}$ Georgi Nadjakov Institute of Solid State Physics, Bulgarian Academy of Sciences, 72 Tzarigradsko Chaussee \\ Blvd., BG-1784 Sofia, Bulgaria, \\ ${ }^{2}$ Centre for Nano and Soft Matter Sciences, P.O.Box 1329, Jalahalli, Bangalore 560 013, India
}

Received October 24, 2015; accepted December 21, 2015; published December 31, 2015

\begin{abstract}
The amplitude-frequency electro-optic (EO) modulation by nanocomposite of room-temperature nematic liquid crystal heptylcyanobiphenyl (7CB) and 3 wt.\% silica nanoparticles (SiNPs) of size $\sim 7 \mathrm{~nm}$, was studied. Thin $(25 \mu \mathrm{m})$ films of SiNPs-7CB soft gel exhibit an extended and flat second-harmonic EO modulation that can be of potential interest for EO applications.
\end{abstract}

Among the soft-matter nanocomposites, the systems consisting of silica nanoparticles (SiNPs) dispersed into nematic liquid crystals (LCs), also known as filled nematics in the most common case, have attracted significant research attention due to their interesting properties and prospective applications in electronic devices utilizing electro-optic (EO), dielectric and memory effects [1-2]. In particular, the EO properties of dispersions of silica nanoparticles (SiNPs) in nematic LCs from the cyano-biphenyl family, have been extensively investigated (e.g., [3-6]).

Here, we study a nanostructured nematic system formed from LC 4-n-heptyl cyanobiphenyl (7CB) doped with SiNPs at a concentration of $3 \mathrm{wt} \%$. The preparation of 25- $\mu$ m-thick SiNPs-7CB nancomposite films was described elsewhere [7]. The dispersion of SiNPs in 7CB was examined by an optical microscope Zeiss NU-2. The EO response of the films upon an alternating-current (AC) electric field was studied by the use of a circularly polarized beam from a $\mathrm{He}-\mathrm{Ne}$ laser (wavelength $\lambda=632.8 \mathrm{~nm}$, power $\sim 1 \mathrm{~mW}$ ) at normal incidence. The AC voltage-dependent light transmittance and frequency response of the SiNPs-7CB films were measured by a photodiode and lock-in technique. For this purpose, a sinusoidal AC voltage from the build-in generator of Stanford Research Systems SR830 DSP lock-in amplifier (additionally amplified through a high voltage amplifier) was applied to the ITO electrodes of the cells with nanocomposite gel films. The sampling signal was acquired by a computer that controls the frequency sweep, in our case in the range $0.1 \mathrm{~Hz}-3 \mathrm{kHz}$. For all measurements reported here, the temperature of the

\footnotetext{
*E-mail: georgibh@issp.bas.bg
}

SiNPs-7CB nanocomposite films was kept at $30^{\circ} \mathrm{C}$. No polarizers were used.

As reported in [7], the room-temperature nematic nanocomposite SiNPs-7CB considered here is a soft gel (where the structure is still flexible [8-9]) with clear evidence for a disordered system. It is generally accepted that SiNPs form aggregates, e.g., the primary SiNPs being single $7 \mathrm{~nm}$ spheres can be clustered in $\sim 40 \mathrm{~nm}$ sized creatures [8]. Rather compact SiNPs agglomerates of larger scale $(\sim 100 \mathrm{~nm})$ can be also possible.

In fact, the prepared nanocomposite gel films displayed by optical microscopy a cloudy-like morphology at macroscopic level (Fig. 1). This texture is changed by varying the AC electric field applied on the films. Actually, the SiNPs dopants lead to formation of a gel network in the SiNPs-7CB [10]. Namely, the random flexible network gives rise to disorders of the LC phase and finite size effects [8, 11-13].
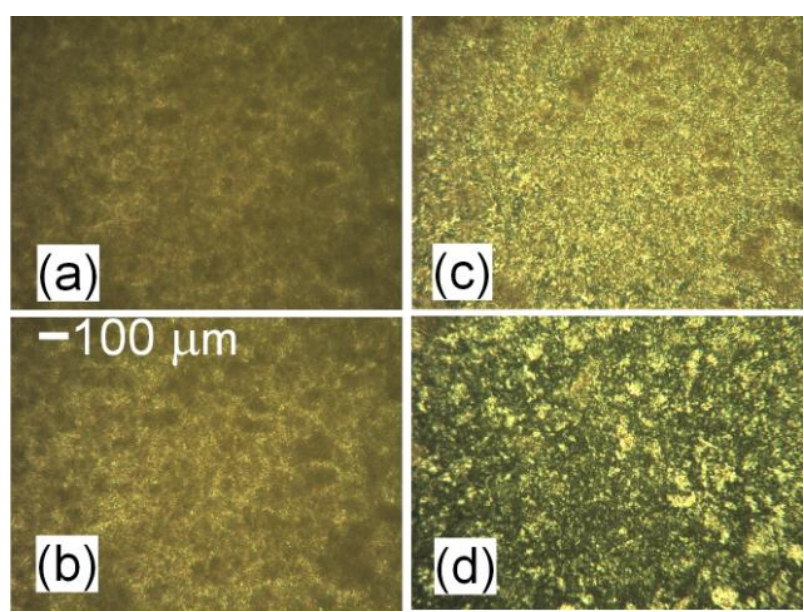

Fig. 1. Micrographs of SiNPs-7CB gel nanocomposite film (with initially planar orientation) upon an $\mathrm{AC}$ electric field $(\mathrm{f}=1 \mathrm{kHz})$ at voltage: 10V (a); 45V (b); 75V (c); 100V (d). 
Because of the presence of the network, domains are formed with random distribution of director orientation of each domain, and the normal structure as well as phase transitions of the partly orientationally ordered mesophases are disturbed. For instance, the effect of quenched random disorder on the phase transitions in $8 \mathrm{CB}$ has been reviewed by Iannacchione [14].

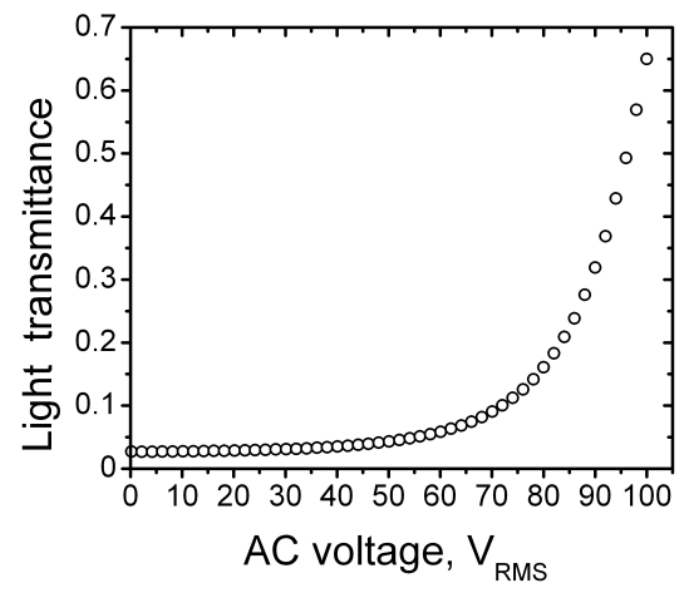

Fig. 2. He-Ne laser beam transmittance of SiNPs-7CB gel film versus the driving $\mathrm{AC}$ voltage $(\mathrm{f}=1 \mathrm{kHz})$.

Due to positive dielectric anisotropy of the host nematic $7 \mathrm{CB}$, upon applying an $\mathrm{AC}$ electric field the optical transmittance of the studied SiNPs-7CB films is increased, since the LC molecules are reoriented along the field direction and the optical scattering is reduced (Fig. 2). This effect is in accordance with the transmittance enhancement, well known for such nanofilled nematics (see, e.g., [1, 15-17]).

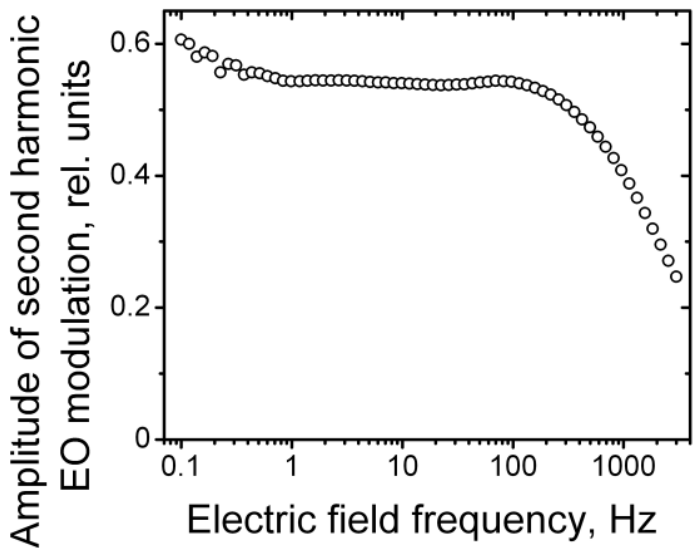

Fig. 3. Frequency spectrum of the amplitude of second-harmonic EO modulation of He-Ne laser beam transmitted through SiNPs-7CB gel film with a thickness of $25 \mu \mathrm{m}$. The amplitude of the driving AC field was kept at $100 \mathrm{~V}_{\mathrm{RMS}}$.
When EO modulation by nanofilled nematic SiNPs7CB was probed, rather flat EO modulation characteristics at the double frequency of the modulated electric field were obtained. Figure 3 presents the amplitude-frequency spectrum of second-harmonic EO modulation of transmitted laser light through such a gel film upon driving voltage $100 \mathrm{~V}$. As seen, this spectrum, i.e., the amplitude of the dielectric oscillations versus the frequency of the applied electric field, is flat in a certain frequency range $(\sim 1 \mathrm{~Hz}-100 \mathrm{~Hz})$. Actually, this frequency dependence is extended towards higher frequencies in comparison to that of the host 7CB nematic. For the latter, the corresponding second-harmonic EO modulation characteristic curve follows the inverse frequency dependence [18]. We have to point out that no such plateau was observed for the first- harmonic EO modulation (the modulation at the fundamental frequency of the driving AC field) by SiNPs-7CB under the same conditions.

(a)
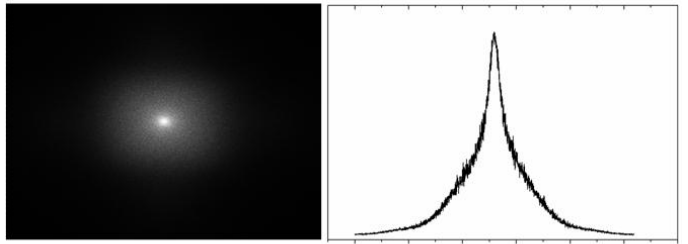

(b)
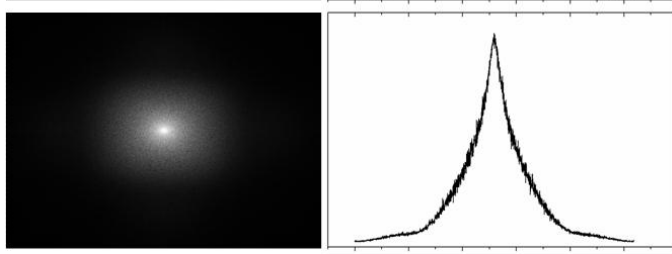

(c)
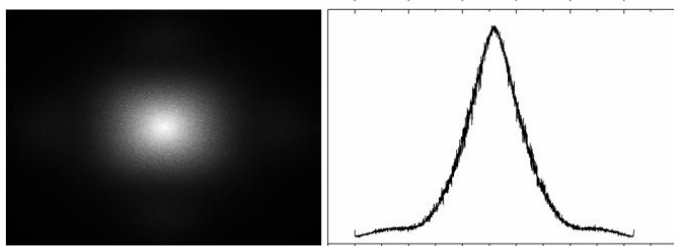

(d)
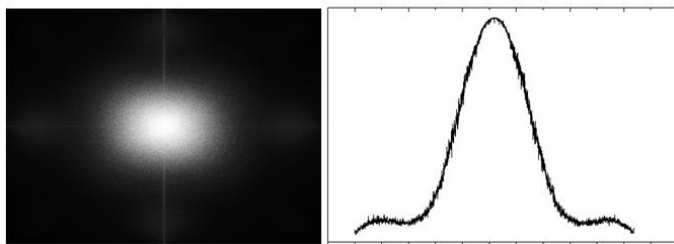

Fig. 4. Left: 2D-FFT of the images from Fig. 1; Right: the corresponding Fourier-amplitude profiles.

This specific extension of the EO frequency-modulation response towards the higher frequencies can be explained with the role of the small structural domains formed in the nanostructured nematic. The small monodomains in SiNPs-7CB nanocomposite films can follow the electric field oscillations at higher frequencies. It should be noted 
that these domains take a significant part in the light scattering of SiNPs-7CB nanocomposite, resulting in the smeared threshold seen in the curve of the voltagedependent transmittance of SiNPs-7CB (Fig. 2), in contrast to pure 7CB nematic. The smeared threshold is because the size of monodomains undergoing Freedericksz transition is much smaller. Based on the Freedericksz transition measurements, one can estimate the dimensions of the two subsystems (the bulk and the nanostructured monodomains) arising within the studied SiNPs-7CB films as a consequence of the LC disorder induced by the SiNPs, both in the bulk and nanoscale vicinity of each particle or network of particles [7].

In this context, it is interesting to note the results obtained by Fourier analysis of the microscope images of the SiNPs-7CB films (Fig. 1), as depending on the voltage applied on the films. The broadening of the corresponding Fourier transforms in the spatial frequency domain (Fig. 4) is indicative of the enhanced role of the smaller domains in the light scattering at higher voltage.

In conclusion, the silica-nanostructured nematic system SiNPs-7CB studied here displays an interesting EO modulation effect of specific flat second-harmonic frequency-modulation EO response, based on the SiNPsinduced disorder, that could be attractive for EO light modulation in an extended frequency range.

INERA EU project Research Potential FP7-316309REGPOT-2012-2013-1 is acknowledged. Work supported by research project DFNI-TO2/18 by the Ministry of Education and Science, National Science Fund of Bulgaria. This study was carried out as a part of IndoBulgarian joint research project DNTS/In-01/4/2013 supported by the Department of Science and Technology, New Delhi and the Ministry of Education and Science, National Science Fund of Bulgaria.

\section{References}

[1] M. Kreuzer, R. Eidenschink, Filled Nematics, in: Liquid Crystals in Complex Geomteries Formed by Polymer and Porous Networks. Eds: G.P. Crawford, S. Zumer, (London: Taylor and Francis 1996), Ch. 15, pp. 307-324.

[2] Y.A. Garbovskiy, A.V. Glushchenko: Liquid Crystalline Colloids of Nanoparticles: Preparation, Properties and Applications, in: Solid State Physics. 62, (Eds.) R.E. Camley, R.L. Stamps (New York, Academic Press, Elsevier 2011) pp. 1-4.

[3] A. Hourri, T.K. Bose, J. Thoen, Phys. Rev. E 63, 051702 (2001).

[4] F.M. Aliev, G.P. Sinha, M. Kreuzer, Mol Cryst. Liq Cryst. 359, 217 (2001).

[5] A.V. Zakharov, J. Thoen, Phys. Rev. E 69, 011704 (2004).

[6] C.V.Lobo, S.K. Prasad, C.V. Yelamaggad, J. Phys.: Condens. Matter 18, 767 (2006).

[7] M.V. Kumar, S.K. Prasad, Y. Marinov, L. Todorova, A.G. Petrov, Mol. Cryst. Liq. Cryst. 610, 51 (2015).

[8] G.S. Iannacchione, J.T. Mang, T.P. Rieker, Phys. Rev. E 58, 5966 (1998).

[9] C.W. Garland, R. Bandyopadhyay, D. Liang, R.H. Colby, J.L. Harden, R.L. Leheny, Phys. Rev. Lett. 94, 107801 (2005).

[10] V. Jayalakshmi, G.G. Nair, S.K. Prasad, J. Phys.: Condens. Matter 19, 226213 (2007)

[11] R.L. Leheny, S. Park, R.J. Birgeneau, J.L. Gallani, C.W. Garland, G.S. Iannacchione, Phys. Rev. E 67, 011708 (2003).

[12] G.S. Iannacchione, S. Park, C.W. Garland, R.J. Birgeneau, R.L. Leheny, Phys. Rev. E 67, 011709 (2003).

[13] A. Roshi, G.S. Iannacchione, P.S. Clegg, R.J. Birgeneau, Phys. Rev. E 69, 031703 (2004).

[14] G.S. Iannacchione, Fluid Phase Equlibria 222-223, 177 (2004).

[15] S. Lee, C. Park, Mol. Cryst. Liq. Cryst. 333, 123 (1999).

[16] T. Bellini, M. Buscaglia, C. Chiccoli, F. Mantegazza, P. Pasini, C. Zannoni, Phys. Rev. Lett. 85, 1008 (2000).

[17] M. Caggioni, A. Roshi, S. Barjami, F. Mantegazza, G.S. Iannacchione, T. Bellini, Phys. Rev. Lett. 93, 127801 (2004).

[18] S.A. Pikin, Strukturnie Prevrashcheniya v Zhidkih Kristallah (in Russian) (Moscow, Nauka 1981) p. 201. 